\title{
Personal identity verification based ECG biometric using non-fiducial features
}

\author{
Marwa A. Elshahed \\ Physics department, Faculty of Women for Arts, Sciences and Education, Ain Shams University, Egypt
}

\begin{tabular}{l} 
Article Info \\
\hline Article history: \\
Received Aug 29, 2019 \\
Revised Jan 5, 2020 \\
Accepted Jan 12, 2020 \\
\hline
\end{tabular}

Keywords:

Biometric

ECG

Fiducial

Non-fiducial

Verification

\begin{abstract}
Biometrics was used as an automated and fast acceptable technology for human identification and it may be behavioral or physiological traits. Any biometric system based on identification or verification modes for human identity. The electrocardiogram (ECG) is considered as one of the physiological biometrics which impossible to mimic or stole. ECG feature extraction methods were performed using fiducial or non-fiducial approaches. This research presents an authentication ECG biometric system using non-fiducial features obtained by Discrete Wavelet Decomposition and the Euclidean Distance technique was used to implement the identity verification. From the obtained results, the proposed system accuracy is $96.66 \%$ also, using the verification system is preferred for a large number of individuals as it takes less time to get the decision.
\end{abstract}

Copyright @ 2020 Institute of Advanced Engineering and Science. All rights reserved.

\section{Corresponding Author:}

Marwa A. Elshahed,

Departement of Physcis, Faculty of Women for Arts, Sciences and Education,

Ain Shams University,

Cairo, Egypt.

Email: lsntl@ccu.edu.tw

\section{INTRODUCTION}

Biometrics is a biological and unique characteristics that identify individuals. Biometrics may be physiological as DNA, Iris, and Fingerprint or behavioral traits as keystroke, gait, and signature. All traits which can be used as biometric should be characterized by Universality, Exclusivity, Permanence, Collectability, Performance, Acceptability and Circumvention [1-3]. The electrocardiogram (ECG) is biological biometric it measures the change of electrical activity of the human heart over time as shown in Figure 1 [4]. The ECG signal consists of three waves start with the electrical P-wave generates from the upper chambers followed by straight-line generates when the blood flows from top chambers to lower chambers. The QRS complex is the next wave which generates from bottom chambers while the last wave is the $\mathrm{T}$ wave from electrical recovery $[2,5,6]$.

ECG features are fiducial and non-fiducial features. Figure 2 shows all fiducial features from the waves which are in the time domain. Non-fiducial features are obtained by using several methods from the transformed domain. These features needless computation time than the fiducial features also it doesn't require to find boundaries of the ECG waveforms which change constantly [7-9]. Two ECG approaches are available based on the used features which are fiducial and non-fiducial approaches as shown in Figure 3. There is no general rule to get the exact locations of ECG signal boundaries because it has continuously variations, so using non-fiducial features is solved this problem [1].

A biometric system may operate as identification or verification schemes. In all systems, the first stage is data collection and preparing the database for the system this process is called the enrollment process as shown in Figure 4. The enrollment process starts with ECG signals collecting from individuals and PIN for each one. ECS signals preprocessing is performed to get more suitable signals then applying one of feature extraction techniques then storing the obtained features in the system database with a PIN for each individual. 


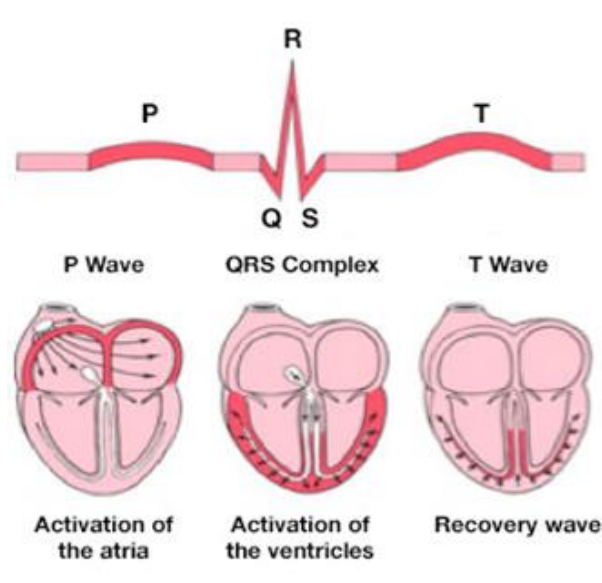

Figure 1. One heartbeat cycle

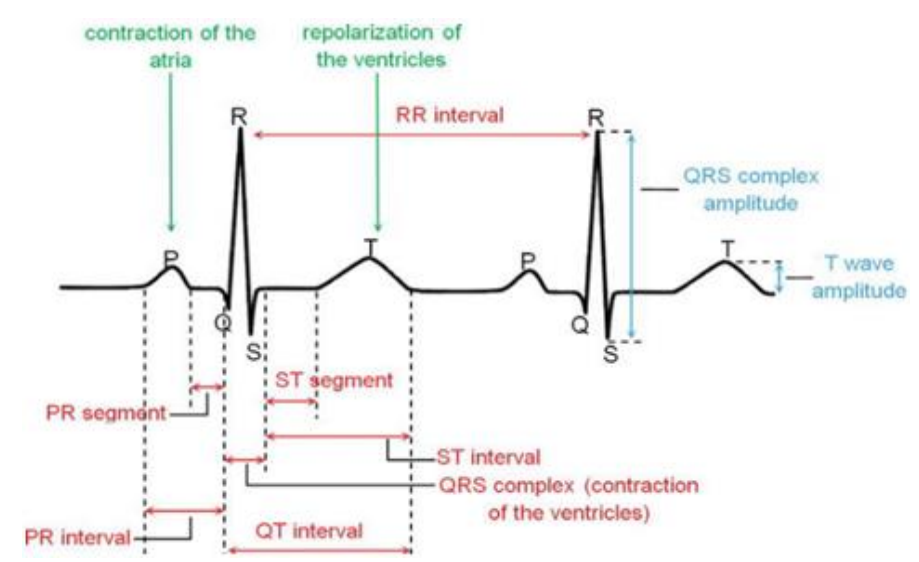

Figure 2. ECG fiducial features [10]

\begin{tabular}{c|ll}
\hline \multirow{2}{*}{ ECG } & $\begin{array}{l}\text { Fiducial } \\
\text { Approches }\end{array}$ & $\begin{array}{l}\text { Time Interval and } \\
\text { Amplitude based } \\
\text { Approaches }\end{array}$ \\
\cline { 2 - 3 } Biometrics & $\begin{array}{l}\text { Non-Fiducial } \\
\text { Approches }\end{array}$ & \begin{tabular}{l} 
Transform Domain Approaches \\
\cline { 2 - 2 }
\end{tabular} \\
\cline { 2 - 3 } & Model Based Approaches \\
\hline
\end{tabular}

Figure 3. ECG biometric approaches

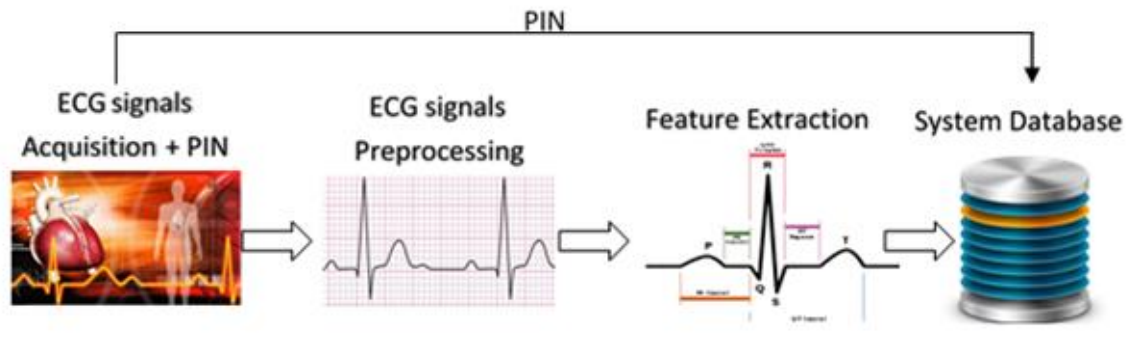

Figure 4. Enrollment process

In an identification system, it starts with the signal acquisition from individual and the same steps used before in the enrollment stage repeated exactly in preprocessing and feature extraction process then comparing between the obtained features with that stores in the database to get the individual identity. it doesn't need identity claimed and considered as one to many matching because it searches on all the database and gets who the user's identity or non-identified as shown in Figure 5. In the verification system, the same as in the enrollment process in ECG signal preprocessing and feature extraction techniques is exactly repeated. The verification system needs identity claimed and considered as one to one matching then getting the decision yes if the features are matching and no for not matching so its result is performed quickly and accurate as shown in Figure 6.

Most of the biometric systems used identification schemes with fiducial and non-fiducial features, while most of verification biometric systems used fiducial features [11-13]. In [2], the Multimodal Biometric Authentication system is proposed using a combination of ECG and Fingerprint together. Fiducial features were extracted from ECG signals after preprocessing also converting the extracted fingerprint to the transformed domain using DWT then extract only two features. The decision was getting from the final score which calculated from matching ECG and fingerprint traits. A lot of parameters were calculated for the system efficiency moreover, the number of subjects is not clear. 


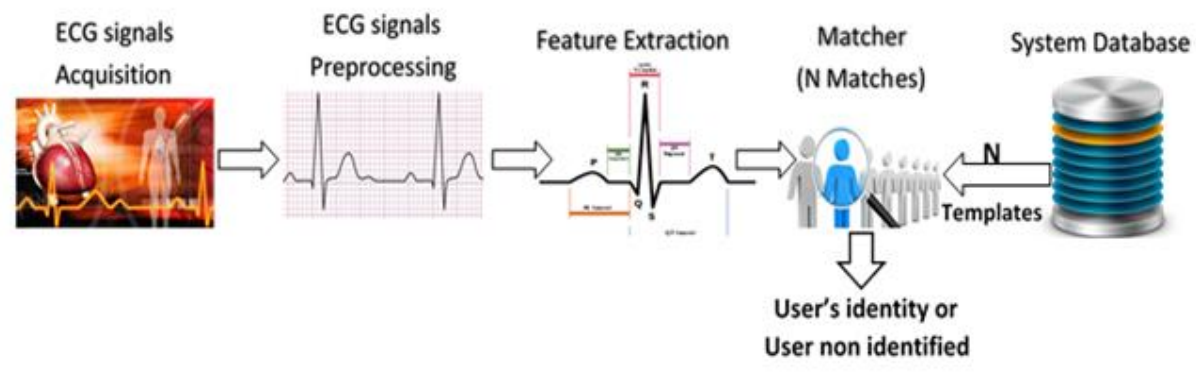

Figure 5. Identification biometric system

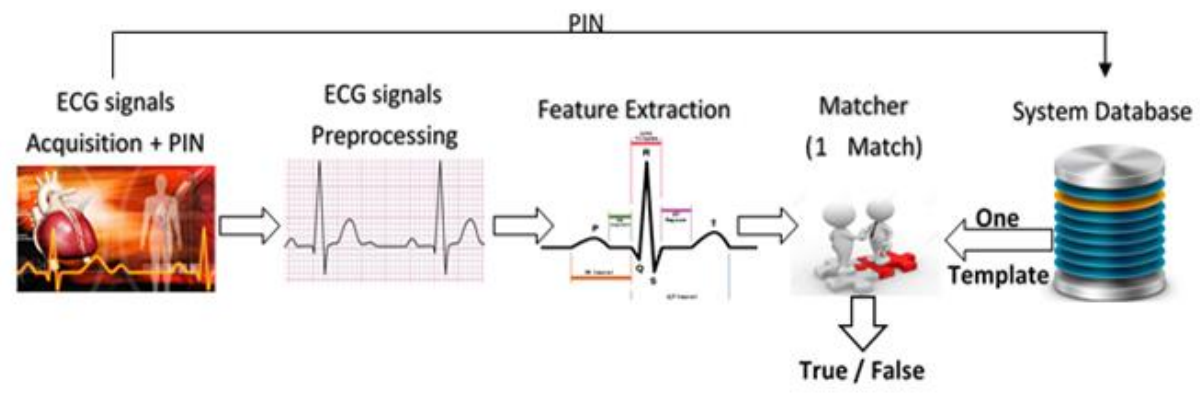

Figure 6. Verification biometric system

The authors in [14], Proposed an Authentication Biometric system which based on the fiducial features directly after preprocessing, they used 73 subjects in their experiment. The Euclidean distance was used for matching. Then they extended their unimodal framework based on ECG trait only to be a multimodal biometric system based on ECG and Face or ECG and fingerprint using score fusion technique. A verification biometric system was proposed in [15], they used only 15 subjects, RR intervals were detected from ECG signals then applying DCT to get the required features. The obtained accuracy is $97.78 \%$ after using the correlation to get the decision.

In [16], ECG Biometric Verification system was proposed by using PCA, they used 8 subjects only in their experiment. They used fiducial features using PCA and DWT for non-fiducial features. The authors in [17], they get the signal features using the Hjorth Descriptor and Sample Entropy (SampEn). Support Vector Machine (SVM) classifier was used for authentication. The obtained accuracy is 93.8\% from Hjorth Descriptor Compared to SampEn. In [18], ECG based biometric system was designed. ECG dataset of 55 users was created during four months to study the ECG-based biometrics stability. He studied the performance of ECG as a biometric trait in short and long terms and found that more needing studies towards improving the long-term ECG biometric systems performance.

The authors in [19] proposed ECG biometric algorithm using 184 subjects from different datasets. They obtained $98.33 \%$ accuracy using a random forest classifier and $96.31 \%$ accuracy using the wavelet distance measure algorithm while the two classifiers together give accuracy 99.52\%. In [20], a non-fiducial ECG biometric verification system was proposed using kernel methods for 52 subjects with Discrete Wavelet Transform (DWT) for denoising. They studied the performance of using Support Vector Machine (SVM) and the Linear Discriminant Analysis (LDA). A two-lead ECG signals biometric verification was proposed in [21], autocorrelation feature extraction method was used in conjunction with different techniques for reduction with different window lengths from short and long-term recordings. The results show that the recognition rates affected by the recording and the window lengths. The objective of this research is to apply a verification biometric system using non-fiducial features with Euclidean distance matching methodology.

\section{RESEARCH METHOD}

The proposed biometric authentication system consists of three main steps pre-processing, feature extraction and reduction using DWT, and matching process, as shown in Figure 7. 


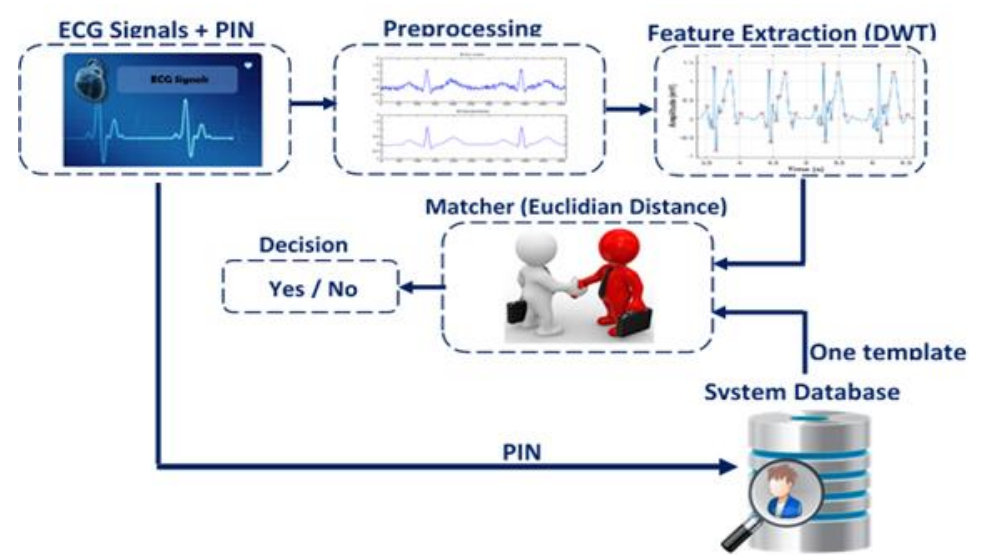

Figure 7. Block diagram of the proposed system

\subsection{Datasets description}

In this research, 90 subjects are used from two datasets. The first dataset is ECG-ID Database while the second is the MIT-BIH Arrhythmia Database [22]. The number of test subjects used in the proposed system is 72 subjects includes 11 unauthorized subjects.

\subsection{Pre-processing}

The first dataset has filtered signals so it used directly while the Butterworth filter was applied on signals from the second dataset. The Pan and Tompkins algorithm was applied for $\mathrm{R}$ peak detection [23]. The cycle length was fixed at 200 samples because features vectors must have an equal length for all subjects. A normalization process into the range from 0 to 1 is applied to the amplitude of all points for each R-R cycle.

\subsection{Feature extraction}

Discrete Wavelet Decomposition was applied as a feature extraction method in each R-R cycle by using Daubechies wavelets (db8) with five-level decomposition as shown in Figure 8. Selecting the Daubechies family in this work depending on its shape and energy spectrum which close to the ECG signal while selecting db8 from previous work which gets good results than others. The total coefficients number after decomposition is 272 as shown in Figure 8. Figure 9. Shows the selected R-R interval and Figure 10 shows that the wavelet coefficients after 100 are approximately closed to zero so those coefficients are neglected ( $\mathrm{d} 1$ and $\mathrm{d} 2)$ and the other coefficients (104) were taken in our experiment.

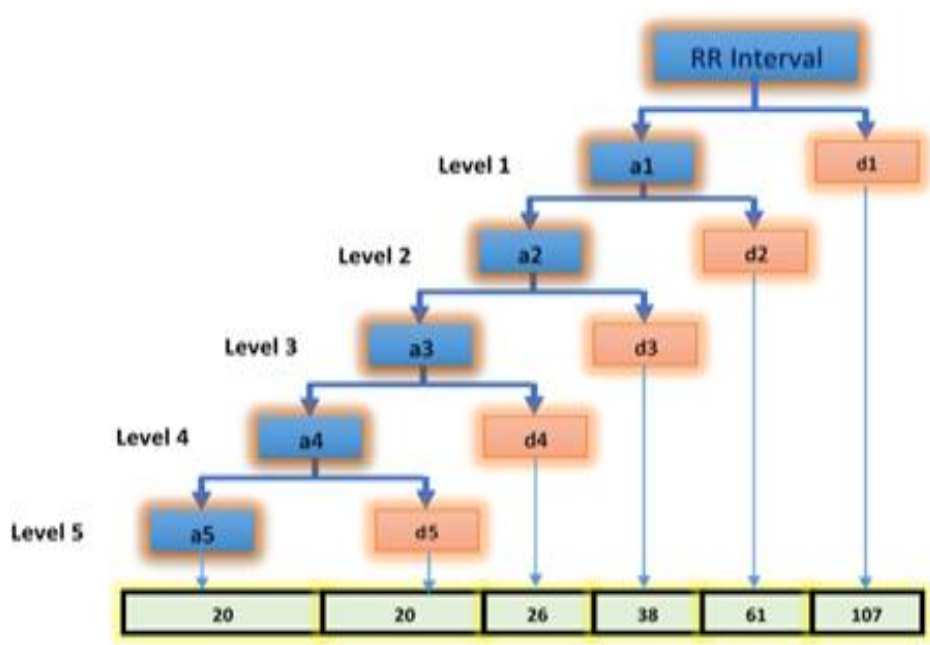

Figure 8. The 5-level discrete wavelet decomposition using Daubechies wavelets 'db8' 


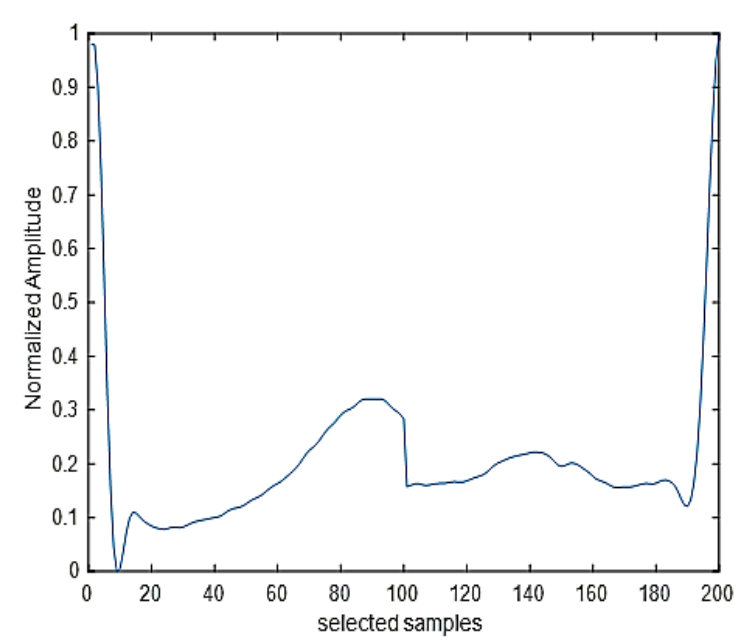

Figure 9. The selected R-R interval

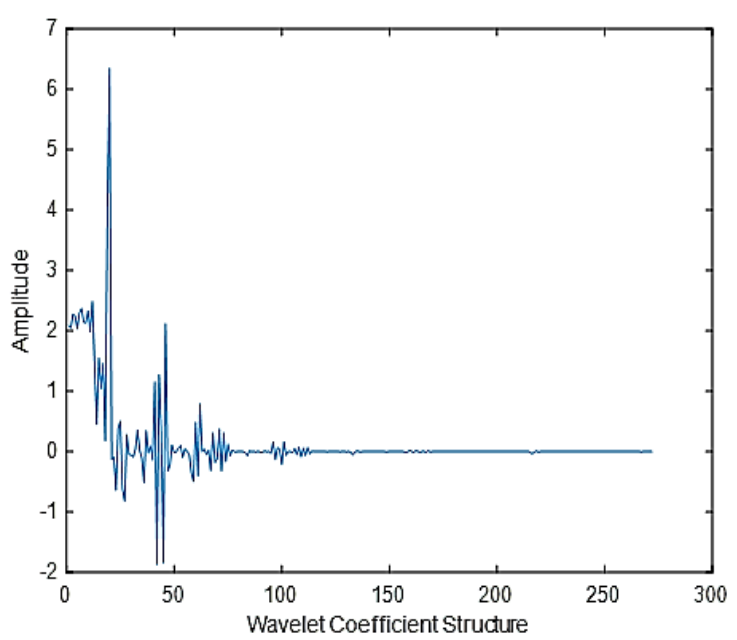

Figure 10. Wavelet coefficients of the selected R-R interval

\subsection{Authentication strategy}

The Euclidean distance algorithm is used for verification decisions. Euclidean distance calculations are performed exactly as follows:

Let $\mathrm{P}(\mathrm{i})$ is the stored pattern feature matrix of size: $m \times d$ :

$$
P^{(i)}=\left[\begin{array}{ccc}
f_{1,1} & \cdots & f_{1, d} \\
\vdots & \ddots & \vdots \\
f_{m, 1} & \cdots & f_{m, d}
\end{array}\right]
$$

If $\mathrm{N}$ is the number of subjects so there where $\mathrm{N}$ stored pattern matrices $\mathrm{P}(\mathrm{i}) . \mathrm{P}^{\prime}(\mathrm{i})$ is the sample features matrix defined as:

$$
P^{\prime(i)}=\left[\begin{array}{ccc}
f^{\prime}{ }_{1,1} & \cdots & f^{\prime}{ }_{1, d} \\
\vdots & \ddots & \vdots \\
f^{\prime}{ }_{m, 1} & \cdots & f^{\prime}{ }_{m, d}
\end{array}\right]
$$

The distance between the attributes of a sample and features matrix of an individual $\mathrm{i}$ is statistically computed using:

$$
d^{(i)}=\left[\begin{array}{ccc}
\left|f_{1,1}-f^{\prime}{ }_{1,1}\right| & \cdots & \left|f_{1, d}-f^{\prime}{ }_{1, d}\right| \\
\vdots & \ddots & \vdots \\
\left|f_{m, 1}-f^{\prime}{ }_{m, 1}\right| & \cdots & \left|f_{m, d}-f^{\prime}{ }_{m, d}\right|
\end{array}\right]
$$

The distance score for an individual is calculated from the sum of Euclidean distances between feature attributes as:

$$
s_{d}^{i}=\sum_{k=1}^{m}\left|f_{k, d-} f^{\prime}{ }_{k, d}\right|
$$

The mean of distance score for an individual (i) should be calculated due to the variations in ECG data set signals as follows:

$$
s^{i}=\frac{1}{d} \sum_{j=1}^{m} s_{j}^{i}
$$

Small distance score value indicates good matching while large distance score value indicates poor matching [14]. To get an accurate decision a threshold is calculated from the distances of the database and the sample feature matrix for each tested individual. 


\section{EXPERIMENT AND RESULTS}

90 subjects are used in the enrolment process to create the system database. Daubechies wavelet 'db8' as a feature extraction method is used. The Euclidean distance algorithm is used as a matching algorithm. The performance of the proposed ECG verification system is estimated by calculating some metrics as the accuracy or verification rate.

$$
\text { verification rate }(\%)=\frac{\text { Total number of correctly samples } N c}{\text { Total number of test samples } N t}
$$

Besides, some metrics are used such as recall, precision, and F-score to evaluate the proposed system performance (6).

$$
\begin{aligned}
& \text { Sensitivity }(\%)=\frac{\mathrm{TP}}{\mathrm{TP}+\mathrm{FN}} \times 100 \\
& \text { Specificity }(\%)=\frac{\mathrm{TN}}{\mathrm{FP}+\mathrm{TN}} \times 100 \\
& \text { Precision }(\%)=\frac{T P}{T P+F p} * 100 \\
& F-\text { score }=\frac{2 * \text { Sensitivity } * \text { Precision }}{(\text { Sensitivity }+ \text { Precision })}
\end{aligned}
$$

Where, TP denotes the number of true positive samples, TN denotes the number of true negative samples, FP denotes the number of false-positive samples and FN denotes the number of false-negative samples [24, 25]. The number of test subjects used in the proposed system is 72 subjects includes 11 unauthorized subjects. The following Table 1 summarizes the performance of the system.

Table 1. The Performance of the proposed system

\begin{tabular}{cc}
\hline Parameters & Percent $(\%)$ \\
\hline Verification Rate & 94.44 \\
Sensitivity & 95.08 \\
Specificity & 90.9 \\
Precision & 98.3 \\
F- Score & 96.66 \\
\hline
\end{tabular}

\section{CONCLUSION}

Some biometric traits could be imitated but the ECG signal is considered as a real-time trait that indicates that the person is alive and present by himself. A verification biometric system based ECG signal is proposed in this research. Daubechies wavelet ' $\mathrm{db} 8$ ' as a feature extraction method is used and the Euclidean distance algorithm for verification decision. 90 subjects are used to build the system database, 72 subjects for testing our verification biometric system. The system performance is evaluated by calculating some metrics. The obtained f-Score is $96.66 \%$. The proposed system is more suitable for a large number of subjects which gives its decision in a very short time with good accuracy due to using the verification approach based on non-fiducial features.

\section{REFERENCES}

[1] Zeeshan Hassan, Syed Omer Gilani and Mohsin Jamil, "Review of Fiducial and Non-Fiducial Techniques of Feature Extraction in ECG based Biometric Systems," Indian Journal of Science and Technology, vol. 9, no. 21, Jun. 2016. DOI: $10.17485 / \mathrm{ijst} / 2016 / \mathrm{v} 9 \mathrm{i} 21 / 94841$

[2] Manjunathswamy B. E., Appaji M Abhishek, Thriveni J, Venugopal K R, and L. M. Patnaik, "Multimodal Biometric Authentication using ECG and Fingerprint," International Journal of Computer Applications (0975-8887), vol. 111, no. 13, Feb. 2015

[3] Ian McAteer, Ahmed Ibrahim, Guanglou Zheng, Wencheng Yang and Craig Valli, "Integration of Biometrics and Steganography: A Comprehensive Review," Technologies, vol. 7, no. 34, 2019. DOI:10.3390/technologies7020034

[4] VPS G4, "Principles of ECG," Vascular Positioning System G4, Vascular Positioning Technology Education, [Online]. Available: http://www.arrowvascular.com/vpseducation/pre-trial/principles-of-ecg.html. 
[5] Ahmed Younes Shdefat, Moon-Il Joo, Sung-Hoon Choi, Hee-Cheol Kim, "Utilizing ECG Waveform Features as New Biometric Authentication Method," International Journal of Electrical and Computer Engineering (IJECE), vol. 8, no. 2, pp. 658 665, Apr. 2018. DOI: 10.11591/ijece.v8i2.pp658-665.

[6] Vanessa Novaes Barros, "The heart cycle: review," MOJ Women's Health, vol. 8, no. 1, 2019.

[7] Woo-Hyuk Jung 1 and Sang-Goog Lee, "ECG Identification Based on Non-Fiducial Feature Extraction Using Window Removal Method," MDPI journal, vol. 7, no. 11, Nov. 2017. DOI: 10.3390/app7111205.

[8] Mohamad O. Diab, Alaa Seif, Mohamad El-Abed and Maher Sabbah, "Individual Identification Using ECG Signals," Journal of Computer and Communications, vol. 8, pp. 74-80, 2018. DOI: 10.4236/jcc.2018.61008.

[9] Di Wang, Yujuan Si, Weiyi Yang, Gong Zhang and Jia Li, "A Novel Electrocardiogram Biometric Identification Method Based on Temporal-Frequency Autoencoding," Electronics, 2019. DOI: 10.3390/electronics8060667.

[10] Țarălungă Dragoș Daniel and Mihaela Neagu, "Cancelling Harmonic Power Line Interference in Biopotentials," book chapter, Intech Open, 2018. DOI: 10.5772/intechopen.74579.

[11] Pankaj Sareen, "Biometrics-Introduction, Characteristics, Basic technique, its Types and Various Performance Measures," International Journal of Emerging Research in Management \& Technology, vol. 3, no. 4, 2014.

[12] Sushma Jaiswal, Sarita Singh Bhadauria and Rakesh Singh Jadon, "Biometric: Case Study," Journal of Global Research in Computer Science, vol. 2, no. 10, Oct. 2011.

[13] Ahmed M. Hamad, Rasha Salah Elhadary, Ahmed Omar Elkhateeb, "Multimodal Biometric Identification Using Fingerprint, Face and Iris Recognition," International Journal of Information Science and Intelligent System, 2014.

[14] Yogendra Narain Singh, S. K. Singh, "Evaluation of Electrocardiogram for Biometric Authentication," Journal of Information Security, vol. 3, no. pp. 39-48, 2012.

[15] Ahmed F. Hussein, Abbas K. AlZubaidi, Ali Al-Bayaty and Qais A. Habash, "An IoT Real-Time Biometric Authentication System Based on ECG Fiducial Extracted Features Using Discrete Cosine Transform," arXiv: 1708.08189, 2017.

[16] Jameel Kadhim Abed and Nahrain N. Abd, "ECG Biometric Verification by using PCA," International Journal of Engineering Trends and Technology (IJETT), vol. 65, no. 1, Nov. 2018.

[17] Sugondo Hadiyoso, Suci Aulia and Achmad Rizal, "One-Lead Electrocardiogram for Biometric Authentication using Time Series Analysis and Support Vector Machine," (IJACSA) International Journal of Advanced Computer Science and Applications, vol. 10, no. 2, 2019.

[18] Nikita Samarin, "A Key to Your Heart: Biometric Authentication Based on ECG Signals," Project Report Computer Science School of Informatics University of Edinburgh, 2018.

[19] Robin Tan and Marek Perkowski, "Toward Improving Electrocardiogram (ECG) Biometric Verification using Mobile Sensors: A Two-Stage Classifier Approach,” Sensors, vol. 17, no. 2 2017. DOI: 10.3390/s 17020410.

[20] M Hejazi, SAR Al-Haddad, YP Singh, SJ Hashim and AFA Aziz, "ECG biometric authentication based on nonfiducial approach using kernel methods," Digital Signal Processing, vol. 52, pp. 72-86, May 2016.

[21] M Hejazi, SAR Al-Haddad, SJ Hashim, AFA Aziz and YP Singh, "Feature level fusion for biometric verification with two-lead ECG signals," IEEE 12th International Colloquium on Signal Processing \& Its Applications(CSPA), pp. 54-59, 2016.

[22] "PhysioBank ATM," PhysioNet, [Online] Available: https://archive.physionet.org/cgi-bin/atm/ATM

[23] Jiapu Pan and Willis j. Tompkins, "A Real-Time Qrs Detection Algorithm," IEEE Transactions on Biomedical Engineering, vol. 32, no. 3, Mar. 1985.

[24] Renuka Joshi, "Accuracy, Precision, Recall \& F1 Score: Interpretation of Performance Measures," 2016. [Online]. Available: https://blog.exsilio.com/all/accuracy-precision-recall-f1-score-interpretation-of-performance-measures/ [Accessed 5/8/2019].

[25] Afzal Godil, Roger Bostelman, Will Shackleford, Tsai Hong and Michael Shneier, "Performance Metrics for Evaluating Object and Human Detection and Tracking Systems," National Institute of Standards and Technology, Jul. 2014. 